\title{
Geometric Singularities of the Stokes Problem
}

\author{
Nejmeddine Chorfi \\ Department of Mathematics, College of Science, King Saud University, P.O. Box 2455, Riyadh 11451, Saudi Arabia
}

Correspondence should be addressed to Nejmeddine Chorfi; nejmeddine.chorfi@yahoo.com

Received 11 November 2013; Accepted 12 December 2013; Published 6 January 2014

Academic Editor: Bessem Samet

Copyright (C) 2014 Nejmeddine Chorfi. This is an open access article distributed under the Creative Commons Attribution License, which permits unrestricted use, distribution, and reproduction in any medium, provided the original work is properly cited.

When the domain is a polygon of $\mathbb{R}^{2}$, the solution of a partial differential equation is written as a sum of a regular part and a linear combination of singular functions. The purpose of this paper is to present explicitly the singular functions of Stokes problem. We prove the Kondratiev method in the case of the crack. We finish by giving some regularity results.

\section{Introduction}

The regularity of the solution of a partial differential equation depends on the geometry of the domain even when the data is smooth. Indeed, for each corner of the polygonal domain a countable family of singular functions can be defined, which depends only on the geometry of the domain. Then the solution of the equation can be written as the sum of a finite number of singular functions multiplied by appropriate coefficients and of a much more regular part. We refer to Kondratiev [1] and Grisvard [2] for their description.

The purpose of our work is to study the singularities of the Stokes equation and the behavior of the solution in the neighborhood of a corner of a polygonal domain of $\mathbb{R}^{2}$. We are interested to nonconvex domains; we assume that there exists an angle equal either to $3 \pi / 2$ or to $2 \pi$ (case of the crack). Handling the singular function is local process, so that there is no restriction to suppose that the nonconvex corner is unique; see Dauge [3]. We deduce the singular function of the velocity from those of the bilaplacian problem with a homogenous boundary conditions by applying the curl operator. We prove the Kondratiev method in the case of the crack. The singularities of the pressure are done by integration from the singular functions of the velocity near the corner.

To approach these problems by a numerical method, we need to take into account the singular functions. Several numeric methods have been proposed in this context; see [49]. Since the singular functions are developed for the Stokes problem in this paper, we intend in future work to implement Strang and Fix algorithm, see [10], by the mortar spectral element method. It will be an extension of a work done on an elliptic operator $[11,12]$.

An outline of this paper is as follows. In Section 2, we present the geometry of the domain and the continuous problem. In Section 3, we give the singular functions and some regularity results. The Kondratiev method is described in Section 4 . Section 5 is devoted to the conclusion.

\section{The Continuous Problem}

We suppose that $\Omega$ is a polygonal domain of $\mathbb{R}^{2}$ simply connected and has a connected boundary $\Gamma . \Gamma$ is the union of vertex $\Gamma_{j}$ for $j \in\{1, \ldots, J\}$; $J$ is positive integer. Let $a_{j}$ be the corner of $\Omega$ between $\Gamma_{j}$ and $\Gamma_{j+1} ; \omega_{j}$ is the measure of the angle on $a_{j}$. We consider the velocity-pressure formulation of the Stokes problem on the domain $\Omega$.

Find the velocity $\mathbf{u}$ and the pressure $p$ such that

$$
\begin{gathered}
-v \Delta \mathbf{u}+\nabla p=\mathbf{f} \quad \text { in } \Omega, \\
\operatorname{div} \mathbf{u}=0 \quad \text { in } \Omega, \\
\mathbf{u}=0 \quad \text { on } \Gamma,
\end{gathered}
$$

where $v$ is the viscosity of the fluid that we suppose a positif constant and $\mathbf{f}$ is the data which represent a density of body forces. Then for $\mathbf{f}$ in $\left[H^{-1}(\Omega)\right]^{2}$, the functional spaces are $\left[H_{0}^{1}(\Omega)\right]^{2}$ for the velocity and $L_{0}^{2}(\Omega)$ for the pressure where

$$
L_{0}^{2}(\Omega)=\left\{q \in L^{2}(\Omega), \int_{\Omega} q(x) d x=0\right\} .
$$


The problem (1) is equivalent to the following variational formulation.

For $\mathbf{f}$ in $\left[H^{-1}(\Omega)\right]^{2}$, find $\mathbf{u}$ in $\left[H_{0}^{1}(\Omega)\right]^{2}$ and $p$ in $L^{2}(\Omega)$ such that for all $\mathbf{v}$ in $\left[H_{0}^{1}(\Omega)\right]^{2}$ and for all $q$ in $L^{2}(\Omega)$.

$$
\begin{gathered}
a(\mathbf{u}, \mathbf{v})+b(\mathbf{v}, p)=\langle f, \mathbf{v}\rangle, \\
b(\mathbf{u}, q)=0,
\end{gathered}
$$

where

$$
\begin{gathered}
a(\mathbf{u}, \mathbf{v})=v \int_{\Omega} \nabla \mathbf{u} \nabla \mathbf{v} d x \\
b(\mathbf{u}, q)=-\int_{\Omega}(\operatorname{div} \mathbf{u}) q d x
\end{gathered}
$$

where the $\langle\cdot, \cdot\rangle$ denotes the duality pairing between $H^{-1}(\Omega)$ and $H_{0}^{1}(\Omega)$. The bilinear form $a(\cdot, \cdot)$ is continuous on the space $\left[H_{0}^{1}(\Omega)\right]^{2} \times\left[H_{0}^{1}(\Omega)\right]^{2}$ and elliptic on $\left[H_{0}^{1}(\Omega)\right]^{2}$; also the bilinear form $b(\cdot, \cdot)$ is continuous and verifies the following inf-sup condition (see $[13,14])$ : there exists a nonnull positive constant $\beta$ such that

$$
\forall q \in L_{0}^{2}(\Omega), \quad \sup _{\mathbf{v} \in\left[H_{0}^{1}(\Omega)\right]^{2}} \frac{b(\mathbf{v}, q)}{\|\mathbf{v}\|_{\left[H^{1}(\Omega)\right]^{2}}} \geq \beta\|q\|_{L^{2}(\Omega)} .
$$

Then we conclude [15] that, for all $\mathbf{f}$ in the space $\left[H^{-1}(\Omega)\right]^{2}$, the problem (3) has a unique solution $(\mathbf{u}, p)$ in $\left[H_{0}^{1}(\Omega)\right]^{2} \times$ $L_{0}^{2}(\Omega)$. This solution verifies the following stability condition:

$$
\|\mathbf{u}\|_{\left[H^{1}(\Omega)\right]^{2}}+\beta\|p\|_{L^{2}(\Omega)} \leq C\|\mathbf{f}\|_{\left[H^{-1}(\Omega)\right]^{2}},
$$

where $C$ is a positive constant.

We refer to the work of Pironneau [16] for the mathematical modeling of problems resulting from fluids mechanics and Girault and Raviart [17] for the mathematical analysis of the Navier-Stokes equations.

\section{Singular Functions and Regularity Results}

We recall that, in an open simply connected of $\mathbb{R}^{2}$, the condition of incompressibility $\operatorname{div}(\mathbf{u})=0$ induces the existence of a stream function $\varphi$ in the space $H_{0}^{2}(\Omega)$ such that

$$
\mathbf{u}=\operatorname{curl}(\varphi)
$$

It thus brings to study the regularity of the function $\varphi$, solution of Dirichlet problem for bilaplacian:

$$
\begin{aligned}
-v \Delta^{2} \varphi & =\operatorname{curl}(\mathbf{f}), \quad \text { in } \Omega, \\
\varphi & =0, \quad \text { on } \Gamma, \\
\frac{\partial \varphi}{\partial n} & =0, \quad \text { on } \Gamma .
\end{aligned}
$$

The regularity of the solution of the problem is related to the geometry of the domain and its behavior is local. Let $g=$ curl(f). We know, see Cattabriga [18] and Ladyzhenskaya [14], the following theorem.
Theorem 1. For $g$ in the space $H^{s}(\Omega)$, where $s \geq-2$, then

$$
\varphi \in H^{s+4}(\Omega \backslash \mathscr{V})
$$

where $\mathscr{V}$ is the union of neighborhoods $V_{j}$ of $a_{j}$ for $j \in$ $\{1, \ldots, J\}$.

To study the function $\varphi$ in the neighborhood of a fixed vertex $a_{j}, j \in 1, \ldots, J$, it is convenient to introduce the polar coordinates $(r, \theta)$, centered at $a_{j}$. We start by enunciating the characteristic equation of bilaplacian:

$$
\sin ^{2} \omega_{j} z=z^{2} \sin ^{2} \omega_{j} .
$$

Then we stated the following theorem. We refer to ([17], Chapter 7 Theorem 7.2.1.12) and Kondratiev [1] for the proof.

Theorem 2. Let $\omega_{j}$ in $] 0,2 \pi\left[\right.$. For all $g$ in $H^{s}(\Omega), s \geq-2$, the solution $\varphi$ of the problem (8) is written as

$$
\varphi=\varphi_{r}+\varphi_{s}
$$

where $\varphi_{r}$ is in the space $\left(H^{s+4}(\Omega) \cap H_{0}^{2}(\Omega)\right)$.

We set

$$
\begin{gathered}
\tau_{k}(r, \theta)=r^{1+z_{k}} \psi_{k}(\theta), \\
\mu_{k}(r, \theta)=r^{1+\widehat{z}_{k}}\left(\sigma_{k}(\theta)+\log (r) \eta_{k}(\theta)\right) .
\end{gathered}
$$

$\varphi_{s}$ is given by

$$
\varphi_{s}=\sum_{0<\operatorname{Re}\left(z_{k}\right)<s+2} \lambda_{k} \tau_{k}(r, \theta)+\sum_{0<\operatorname{Re}\left(\widehat{z}_{k}\right)<s+2} \hat{\lambda}_{k} \mu_{k}(r, \theta),
$$

where $\lambda_{k}$ and $\hat{\lambda}_{k}$ are real constants, $\psi_{k}, \sigma_{k}$, and $\eta_{k}$ belong to the vectorial space $\mathscr{C}^{\infty}\left(\left[0, \omega_{j}\right]\right) \cap H_{0}^{2}\left(\left[0, \omega_{j}\right]\right)$ of finite dimension, $z_{k}$ and $\widehat{z}_{k}$ are, respectively, the simple and double roots of (10) in the band $0<\operatorname{Re}\left(z_{k}\right)<s+2$, excepting 1 if $\omega_{j} \neq \operatorname{tg}\left(\omega_{j}\right)$, without exception if $\omega_{j}=\operatorname{tg}\left(\omega_{j}\right)$.

We called $\omega_{e}$ the unique solution of equation $\omega=\operatorname{tg}(\omega)$, in the $] 0,2 \pi\left[\left(\omega_{e} \simeq 1.430297 \pi\right)\right.$. We prove, see ([19], chapter $3, \S 3.3)$, that $z$ is a double root of (10) if and only if $z=0$ or $z= \pm \sqrt{\left(1 / \sin \omega_{j}^{2}\right)-\left(1 / \omega_{j}^{2}\right)}$. Hence the sufficient condition on the angle $\omega$ for a double root is

$$
\sin \left(\sqrt{\frac{\omega_{j}^{2}}{\left(\sin \omega_{j}\right)^{2}}-1}\right)= \pm \sqrt{\left(1-\frac{\left(\sin \omega_{j}\right)^{2}}{\omega_{j}^{2}}\right)},
$$

Then, if $\omega_{j}$ is not a solution of (14), (10) takes the following simplified form:

$$
\varphi_{s}(r, \theta)=\sum_{0<\operatorname{Re}\left(z_{k}\right)<s+2} \lambda_{k} \tau_{k}(r, \theta) .
$$

In the following we suppose that $\Omega$ has a unique vertex $a$ where $\omega$ is equal to $3 \pi / 2$ or $2 \pi$ and the other angles are $\pi / 2$. 
We introduce $V$ as a neighborhood of $a$. All these angles are different of $\omega_{e}$.

In the case where $\omega=3 \pi / 2$ and $s=-1$, (10) has two real simple roots in the band $0<\operatorname{Re}(z)<1$. We apply the Newton method to approximate those roots $z_{1} \simeq 0.544484$ and $z_{2} \simeq 0.908529$. The functions $\tau_{1}$ and $\tau_{2}$ are written as follows:

$$
\begin{gathered}
\tau_{1}(r, \theta)=r^{1+z_{1}} \psi_{1}(\theta), \\
\tau_{2}(r, \theta)=r^{1+z_{2}} \psi_{2}(\theta),
\end{gathered}
$$

with

$$
\begin{aligned}
\psi_{i}(\theta)= & \left(\left(z_{i}-1\right)^{-1} \sin \left(\frac{3\left(z_{i}-1\right) \pi}{2}\right)-\left(z_{i}+1\right)^{-1}\right. \\
& \left.\quad \times \sin \left(\frac{3\left(z_{i}+1\right) \pi}{2}\right)\right) \\
& \times\left(\cos \left(\left(z_{i}-1\right) \theta\right)-\cos \left(\left(z_{i}+1\right) \theta\right)\right) \\
& -\left(\left(z_{i}-1\right)^{-1} \sin \left(\left(z_{i}-1\right) \theta\right)-\left(z_{i}+1\right)^{-1} \sin \left(\left(z_{i}+1\right) \theta\right)\right) \\
& \times\left(\cos \left(\frac{3\left(z_{i}-1\right) \pi}{2}\right)-\cos \left(\frac{3\left(z_{i}+1\right) \pi}{2}\right)\right) .
\end{aligned}
$$

Proposition 3. For all $\epsilon \geq 0$, the functions $\tau_{i}, i$ in $\{1,2\}$, are belonging to the space $H^{\left(2+z_{i}\right)-\epsilon}(V)$ and are solutions of the problem:

$$
\begin{array}{cc}
\Delta^{2} \tau_{i}=0 & \text { in } V, \\
\tau_{i}=\frac{\partial \tau_{i}}{\partial n}=0 & \text { on } \Gamma \cap \partial V,
\end{array}
$$

where $\Gamma$ is the boundary of $\Omega$.

Proof. Since $\psi_{i}$ is in $\mathscr{C}^{0}(] 0,3 \pi / 2[)$, we find $s$ such that

$$
r^{1+z_{i}} \psi_{i}(\theta) \in H^{s}(V) .
$$

Then we find $(m, q)$ in $\mathbb{N} \times \mathbb{R}, m>s$, and $1<q \leq 2$ such that:

$$
r^{1+z_{i}} \psi_{i}(\theta) \in W_{q}^{m}(V) \subset H^{s}(V) .
$$

From the Sobolev injection theorem, we have $m-2=s-1$. If $r^{1+z_{i}} \psi_{i}(\theta) \in W_{q}^{m}(V)$, then $1+z_{i}>m-2 / q$. Since $1<q \leq$ 2 , we can take only the value $E\left(3+z_{i}\right)=3\left(0<z_{i}<1\right)$. Consequently, $s<\left(2+z_{i}\right)$ for $i \in\{1,2\}$. And by construction, we obtain that $\tau_{i}$, for $i \in\{1,2\}$, is the solution of problem (18).

Corollary 4. For all $f$ in $\left[L^{2}(\Omega)\right]^{2}$, the velocity $\mathbf{u}$ is written as

$$
\mathbf{u}=\mathbf{u}_{r}+\mathbf{u}_{s}
$$

where $\mathbf{u}_{r}$ is in $\left[H^{2}(\Omega) \cap H_{0}^{1}(\Omega)\right]^{2}$ and there exists a constant $\lambda_{i}$ such that:

$$
\mathbf{u}_{s}=\sum_{1 \leq i \leq 2} \lambda_{i} s_{i},
$$

where $s_{i}(r, \theta)=\operatorname{curl}\left(\tau_{i}(r, \theta)\right)$. The functions $s_{i}$ belong to the space $\left[H^{\left(1+z_{i}\right)-\epsilon}(V)\right]^{2}, i$ in $\{1,2\}$, for all $\epsilon>0$.

\section{Kondratiev's Method Case of the Crack}

In the case of the crack there are no known results; we recall the method of kondratiev. We extend this method to the case of crack. We consider the polar coordinates and a truncation function $\chi$ with compact support that does not intersect with the boundary $\Gamma$. We define

$$
G=\left\{r e^{i \theta}, 0<\theta<2 \pi, r>0\right\} .
$$

Let $\psi=\chi \varphi$; then, $\psi$ is in the space $H_{0}^{2}(G)$; with a compact support included in $G$, then $\psi$ is the solution of the problem:

$$
v \Delta^{2} \psi=g \text { in } H^{s}(G),
$$

$$
\psi \in H_{0}^{2}(G) \quad \text { with compact support. }
$$

Definition 5. For $s \geq 0$, one defines the weight Sobolev space:

$$
\begin{gathered}
Z_{2}^{s}(G)=\left\{\psi \in L^{2}(G), r^{-s+|\alpha|} D^{\alpha} \psi \in L^{2}(G),|\alpha| \leq s\right\} \\
Z_{2}^{-1}(G)=\left\{\psi \in \mathscr{D}^{\prime}(G), \psi=\frac{g_{0}}{r}+\partial_{x} g_{1}+\partial_{y} g_{2},\right. \\
\text { such that } \left.g_{0}, g_{1} \text { and } g_{2} \in L^{2}(\Omega)\right\} .
\end{gathered}
$$

Remark 6. We remark that $Z_{2}^{0}(G)=Z^{2}(G)$ and $H_{0}^{s}(G) \subset$ $Z_{2}^{s}(G) \subset H^{s}(G)$.

In the following, we suppose that $g$ is in the space $Z_{2}^{s}(G)$, for $s$ in $\{-1,0\}$. The solution $\psi$ of the problem:

$$
\begin{gathered}
v \Delta^{2} \psi=g \quad \text { in } H^{s}(G), \\
u \in Z_{2}^{2}(G),
\end{gathered}
$$

is with a compact support; then, there exists a real number $R$ such that

$$
\psi(r, \theta)=0, \quad \text { for } r \geq R .
$$

The Kondratiev's method consists to change the variable $r=e^{t}$; then we replace the domain $G$ by the domain $B=$ $\mathbb{R} \times] 0,2 \pi[$, and the weight Sobolev space by the ordinary one. We apply the Fourier transformation relative to the first variable of the problem (26).

Proposition 7. If $\psi$ is in the space $P_{2}^{2}(G)$, the function $v=$ $e^{-t} \psi\left(e^{t} \cos \theta, e^{t} \sin \theta\right)$ belongs to $H^{2}(B)$.

For $s \in\{-1,0\}$, if $g$ belongs to $P_{2}^{s}(G)$, and let $h=$ $e^{3 t} \psi\left(e^{t} \cos \theta, e^{t} \sin \theta\right)$, then the function $e^{-(s+2) t} h$ is in the space $H^{s}(B)$.

Proof. Let $s=-1$. 
Since $g \in P_{2}^{-1}(G)$, then $g=g_{0} / r+\partial_{x} g_{1}+\partial_{y} g_{2}$ with $g_{0}, g_{1}$, and $g_{2}$ in $L^{2}(G)$.

We denote

$$
\begin{gathered}
F(t, \theta)=g\left(e^{t} \cos \theta, e^{t} \sin \theta\right), \\
G_{j}(t, \theta)=g_{j}\left(e^{t} \cos \theta, e^{t} \sin \theta\right), \quad \text { for } j \in\{0,1,2\}, \\
F(t, \theta)=e^{-t} G_{0}+\left(e^{-t} \cos \theta \frac{\partial G_{1}}{\partial t}-e^{-t} \sin \theta \frac{\partial G_{1}}{\partial \theta}\right) \\
+\left(e^{-t} \sin \theta \frac{\partial G_{2}}{\partial t}-e^{-t} \cos \theta \frac{\partial G_{2}}{\partial \theta}\right) .
\end{gathered}
$$

We have

$$
\begin{aligned}
e^{-t} h= & e^{2 t} F(t, \theta)=e^{t} G_{0}+\partial_{t}\left(e^{t} \cos \theta G_{1}+\sin \theta e^{t} G_{2}\right) \\
& +\partial_{\theta}\left(-e^{t} \sin \theta G_{1}+\cos \theta e^{t} G_{2}\right)
\end{aligned}
$$

and if

$$
\begin{gathered}
K_{0}=G_{0}, \\
K_{1}=\cos \theta G_{1}+\sin \theta G_{2}, \\
K_{2}=-\sin \theta G_{1}+\cos \theta G_{2},
\end{gathered}
$$

then we conclude

$$
e^{-t} h=e^{t} K_{0}+\partial_{t}\left\{e^{t} K_{1}\right\}+\partial_{\theta}\left\{e^{t} K_{2}\right\}
$$

since $g_{j} \in L^{2}(G)$ et $e^{t} G_{j} \in L^{2}(B)$, then, $e^{t} K_{j} \in L^{2}(B)$ for $j \in\{0,1,2\}$. So

$$
e^{-t} h \in H^{-1}(B)
$$

We end the proof.

As $\psi$ is the solution of the problem (26), then for $h=e^{3 t} g$ the function $v=e^{-t} \psi\left(e^{t} \cos \theta, e^{t} \sin \theta\right)$ is a solution of the problem:

$$
\begin{array}{r}
\left(D_{t}^{4}-D_{t}^{2}+1\right) v+2\left(D_{t}^{2}+1\right) D_{\theta}^{2} v+D_{\theta}^{4} v=h \\
v(t, 0)=v(t, 2 \pi)=\partial_{t} v(t, 0)=\partial_{\theta} v(t, 2 \pi)=0, \\
v \in H^{2}(B) .
\end{array}
$$

We have $v(t, \theta)=0$ for $t \geq \log (R)=t_{0}$.

The Fourier's transformation on the variable $t$ of the function $v$ is

$$
\widehat{v}(z, t)=\frac{1}{\sqrt{2 \pi}} \int_{\mathbb{R}} e^{-i t z} v(t, z) d t .
$$

$z$ is a complex variable; we denote:

$$
L\left(z, D_{\theta}\right)=\left(z^{4}+2 z^{2}+1\right)+2\left(1-z^{2}\right) D_{\theta}^{2}+D_{\theta}^{4} .
$$

The function $\widehat{v}$ becomes a solution of the problem:

$$
\begin{gathered}
L\left(z, D_{\theta}\right) \widehat{v}=\widehat{h}, \\
\widehat{v}(t, 0)=\widehat{v}(t, 2 \pi)=\partial_{t} \widehat{v}(t, 0)=\partial_{\theta} \widehat{v}(t, 2 \pi)=0 .
\end{gathered}
$$

For all $z$ such that $\operatorname{Im}(z) \geq 0$, the operator $L\left(z, D_{\theta}\right)$ has

$$
p^{4}+2\left(1-z^{2}\right) p^{2}+\left(z^{4}+2 z^{2}+1\right)=0
$$

as a characteristic polynomial. This polynomial function has two roots $p= \pm z$ and $p= \pm i$.

(i) If $z \neq 0$ and $z \neq \pm i$, then the fundamental solutions of the fourth order partial differential equation are

$\sin \theta \operatorname{sh} \theta z, \quad \sin \theta \operatorname{ch} z \theta, \quad \cos \theta \operatorname{ch} \theta z, \quad$ et $\cos \theta \operatorname{sh} z \theta$.

(ii) If $z=0$, the solutions have the form

$$
\sin \theta, \quad \cos \theta, \quad \theta \sin \theta, \quad \text { et } \theta \cos \theta .
$$

(iii) If $z= \pm i$, the solutions have the form

$$
1, \quad \theta, \quad \sin 2 \theta, \quad \text { et } \cos 2 \theta \text {. }
$$

Then, let the following homogeneous problem

$$
\begin{gathered}
\left.L\left(z, D_{\theta}\right) \varphi=0 \text { in }\right] 0,2 \pi[ \\
\varphi(0)=\varphi(2 \pi)=\partial_{t} \varphi(0)=\partial_{\theta} \varphi(2 \pi)=0 .
\end{gathered}
$$

We note $E_{z}=\left\{\varphi_{z} \in H_{0}^{2}(] 0,2 \pi[), \varphi_{z}\right.$ solution of the problem (41)\}.

Proposition 8. The set $E_{z}$ satisfies the following properties:

(1) if $z \neq 0$ and $z \neq \pm i$ where $\operatorname{sh} 2 \pi z \neq 0$, then,

$$
E_{z}=\{0\}
$$

(2) if $z \pm i$, then,

$$
E_{z}=\left\{\varphi_{z} \in H_{0}^{2}(] 0,2 \pi[), \varphi_{z}=\alpha_{z} \sin ^{2} \theta, \alpha_{z} \in \mathbb{C}\right\},
$$

(3) if $z \neq 0, z \neq \pm i$ and $\operatorname{sh} 2 \pi z=0$, which implies that $z$ is equal to $-i(k / 2)$ where $k \neq 0$ and $k \neq 2$, then,

$$
\begin{gathered}
E_{z}=\left\{\varphi_{z} \in H_{0}^{2}([0,2 \pi]), \varphi_{z}=\alpha_{k} g_{k}(\theta)+\beta_{k} h_{k}(\theta),\right. \\
\left.\left(\alpha_{k}, \beta_{k}\right) \in \mathbb{C}^{2}\right\}, \\
g_{k}(\theta)=\sin \left(1+\frac{k}{2}\right) \theta-\left(\frac{k+2}{k-2}\right) \sin \left(-1+\frac{k}{2}\right) \theta, \\
h_{k}=\cos \left(1+\frac{k}{2}\right) \theta-\cos \left(-1+\frac{k}{2}\right) \theta .
\end{gathered}
$$

Proof. If $z \neq 0$ and $z \neq \pm i$,

$$
\varphi=\alpha \sin \partial \operatorname{sh} z \theta+\beta \sin \theta \operatorname{ch} z \theta+\gamma \cos \theta \operatorname{sh} z \theta+\delta \cos \theta \operatorname{ch} z \theta
$$


is solution of the problem (41). Since $\varphi(0)=\varphi(2 \pi)=$ $\partial t \varphi(0)=\partial_{\theta} \varphi(2 \pi)=0$, we obtain the following system:

$$
\begin{gathered}
\delta=0, \\
\gamma \operatorname{sh} 2 \pi z+\delta \operatorname{ch} 2 \pi z=0, \\
\beta=0, \\
\alpha \operatorname{sh} 2 \pi z+\beta \operatorname{ch} 2 \pi z+2 \pi \gamma \operatorname{sh} 2 \pi z+2 \pi \delta \operatorname{ch} 2 \pi z=0,
\end{gathered}
$$

its determinant is equal to $\operatorname{sh}^{2} 2 \pi z$. Then, if $\operatorname{sh} 2 \pi z \neq 0, E_{z}=$ $\{0\}$. Otherwise, the system has the following complexes roots: $z_{k}=-i(k / 2)$ for $k \neq 0$ and $k \neq 2, k \in \mathbb{N}$. The space of solutions of the homogeneous equation is of dimension 2. Indeed, for $z=z_{k}$, we obtain

$$
\varphi=\frac{\alpha}{2} i h_{k}(\theta)+i \gamma\left(\frac{k}{2}-1\right) g_{k}(\theta) .
$$

We check that $\left(h_{k}, g_{k}\right)$ are two independent solutions of the problem (41); then

$$
\int_{0}^{2 \pi}\left|h_{k}\right| d \theta=\int_{0}^{2 \pi}\left|g_{k}\right| d \theta=1 .
$$

This ends the proof of 1 and 3 .

Now we prove property 2 .

If $z= \pm i$

$$
\varphi=\alpha+\beta \theta+\gamma \sin 2 \theta+\delta \cos 2 \theta
$$

is solution of the problem (41), which gives the following system:

$$
\begin{gathered}
\alpha+\delta=0, \\
\alpha+2 \pi \beta+\delta=0, \\
\beta+2 \gamma=0 .
\end{gathered}
$$

The determinant of this system is zero; then the dimension of $E_{z}$ is equal to 1 . Indeed,

$$
\varphi=\alpha(1-\cos 2 \theta)=2 \alpha \sin ^{2} 2 \theta .
$$

This completed the proof.

Remark 9. If

$$
D_{k}=\left\{z_{k}=-i \frac{k}{2}, \text { for } k \neq 0, k \neq 2, k \in \mathbb{N}\right\} .
$$

$D_{k}$ is the set of nonregular values. Since $\widehat{h}$ is analytic for $\operatorname{Im}(z)>-(s+2)$, then $\widehat{v}$ analytically extends across $\{\operatorname{Im}(z)>$ $-(s+2)\} \cap D_{k}$.

Is then

$$
d(z)=\frac{-s h^{2} 2 \pi z}{z^{2}\left(1+z^{2}\right)}, \quad \text { for } z \neq 0, \quad z \neq \pm i
$$

We verify that $d\left(z_{k}\right)=\partial_{z} d\left(z_{k}\right)=0$ and $\partial_{z}^{2} d\left(z_{k}\right) \neq 0$. Hence the multiplicity of $z_{k}$ is 2 for $k \neq 0$ and $k \neq 2 . d( \pm i)=0$, then, $( \pm i)$ are simple solutions.
Theorem 10. (1) The solution $\widehat{v}$ of the problem (36) can be written in the neighborhood of $-i$ :

$$
\widehat{v}(z, \theta)=\frac{\varphi_{1}(\theta)}{(z+i)}+\widehat{w}_{1}(z, \theta),
$$

where $\varphi_{1}$ is the solution of problem (41) with $z=(-i)$ and $w_{1}$ is an analytic function in the neighborhood of $-i$ with values in $H^{2}(] 0,2 \pi[)$.

(2) The solution $\hat{v}$ of the problem (36) is written in the neighborhood of $(-i(k / 2)), k=1$ or $k=3$, as

$$
\widehat{v}(z, \theta)=\frac{\psi_{k}(\theta)}{(z+i(k / 2))^{2}}+\frac{\varphi_{k}(\theta)}{(z+i(k / 2))}+\widehat{w}_{k}(z, \theta),
$$

where $\psi_{k}$ and $w_{k}$ satisfy the same properties as above and $\varphi_{k}$ satisfies:

$$
\begin{gathered}
L\left(z, D_{\theta}\right) \varphi_{k}=2 i k\left(1-\frac{k^{2}}{4}\right) \psi_{k}-2 i k \psi_{k}^{\prime \prime}, \\
\varphi_{k}(0)=\varphi_{k}(2 \pi)=\partial_{t} \varphi_{k}(0)=\partial_{\theta} \varphi_{k}(2 \pi)=0 .
\end{gathered}
$$

Proof. We verified that any solution to the problem (41) is a linear combination of

$$
\begin{gathered}
u_{1}(z, \theta)=\frac{\sin \theta \operatorname{sh} z \theta}{z} \text { for } z \neq 0, \\
u_{1}(0, \theta)=\theta \sin \theta, \\
u_{2}(z, \theta)=\frac{1}{z^{2}+1}\left(\frac{\cos \theta \operatorname{sh} z \theta}{z}-\sin \theta \operatorname{ch} z \theta\right), \\
\text { for } z \neq 0, z \neq \pm i, \\
u_{2}(0, \theta)=\theta \cos \theta-\sin \theta, \\
u_{2}( \pm i, \theta)=\frac{1}{2}\left(\frac{\sin 2 \theta}{2}-\theta\right) .
\end{gathered}
$$

In fact, if $z=-i(k / 2)$ for $k=1$ or $k=3$, we have

$$
\begin{gathered}
u_{1}\left(-i \frac{k}{2}, \theta\right)=-\frac{1}{k} h_{k}(\theta), \\
u_{2}\left(-i \frac{k}{2}, \theta\right)=\frac{2}{k(k+2)} g_{k}(\theta) .
\end{gathered}
$$

Therefore, any solution of the problem (41) is linear combination of $u_{1}$ and $u_{2}$. We also verified that $u_{1}$ and $u_{2}$ are whole solutions with respect to the variable $z$ and if we set

$$
d_{z}=u_{1}(z, 2 \pi) D_{\theta} u_{2}(z, 2 \pi)-u_{2}(z, 2 \pi) D_{\theta} u_{1}(z, 2 \pi),
$$

we note that $d\left(z_{k}\right) \widehat{v}\left(z_{k}, \theta\right)=0$. This implies that $\widehat{v}$ is analytical in the neighborhood of $z_{k}=-i(k / 2)$ for $k$ in $\{1,2,3\}$. (-i) is a single root of $d$; then, $\widehat{v}$ admits a simple pole at $(-i)$. On the other side $-i / 2$ and $(-3 / 2) i$ are roots of multiplicity two of $d$, and poles of multiplicity two of $\widehat{v}$.

In the neighborhood of $(-i), \widehat{v}(z, \theta)=\left(\psi_{1}(\theta) /(z-i)\right)+$ $\widehat{w}_{1}(z, \theta)$, and, for all $z$,

$$
\begin{aligned}
(z-i) \widehat{h} & =L\left(-i, D_{\theta}\right) \widehat{v}(z-i) \\
& =L\left(-i, D_{\theta}\right) \psi_{1}+(z-i) L\left(-i, D_{\theta}\right) \widehat{w}_{1} .
\end{aligned}
$$


Then, for $z=-i$, we have

$$
\begin{gathered}
L\left(-i, D_{\theta}\right) \psi_{1}=0, \\
\psi_{1}(0)=(z-i) \widehat{w}_{1}(z, 0) .
\end{gathered}
$$

Then

$$
\psi_{1}(0)=0
$$

and even

$$
\begin{gathered}
\psi_{1}(2 \pi)=0, \\
\psi_{1}^{\prime}(0)=\lim _{\theta \rightarrow 0} \frac{\psi(\theta)}{\theta}=\lim _{\theta \rightarrow 0} \frac{\widehat{v}(z, \theta)-(z+1) \widehat{w}_{1}(z, \theta)}{\theta}=0,
\end{gathered}
$$

and even for:

$$
\psi_{1}^{\prime}(2 \pi)=0 .
$$

Thus, $\psi_{1}$ is a solution of the problem (41) at $z=-i$.

The expression of $\widehat{v}$ in the neighborhood of $z_{k}=-i(k / 2)$ for $k=1$ and $k=3$ is

$$
\begin{array}{r}
\widehat{v}(z, \theta)=\frac{\psi_{k}(\theta)}{(z+i(k / 2))^{2}}+\frac{\varphi_{k}(\theta)}{(z+i(k / 2))}+\widehat{w}_{k}(z, \theta), \\
\text { for } k=1, k=3 .
\end{array}
$$

Then, for $z$ in the neighborhood of $z_{k}$

$$
\begin{aligned}
\widehat{h}\left(z-z_{k}\right)^{2}= & L\left(z, D_{\theta}\right) \psi_{k}+\left(z-z_{k}\right) L\left(z, D_{\theta}\right) \varphi_{k} \\
& +\left(z-z_{k}\right)^{2} L\left(z, D_{\theta}\right) \widehat{w}_{k} .
\end{aligned}
$$

For $z=z_{k}$, we obtain that $L\left(z_{k}, D_{\theta}\right) \psi_{k}=0$ and we verify that $\psi_{k}$ is solution of the problem (41). And we have:

$$
\begin{aligned}
L\left(z, D_{\theta}\right) \varphi_{k}= & -\frac{L(z, D \theta)+L\left(z_{k}, D_{\theta}\right)}{\left(z-z_{k}\right)} \psi_{k} \\
& +\left(z-z_{k}\right)\left(\widehat{h}-L(z, D \theta) \widehat{w}_{k}\right) .
\end{aligned}
$$

And if $z$ tends to $z_{k}$

$$
L\left(z, D_{\theta}\right) \varphi_{k}=2 i k\left(1-\frac{k^{2}}{4}\right) \psi_{k}-2 i k \psi_{k}^{\prime \prime}
$$

and $\varphi_{k}$ verifies the boundary conditions, which completes the proof.

Remark 11. We remark that the operator $L\left(z, D_{\theta}\right)$ is selfadjoint:

$$
\begin{aligned}
\left\langle L \psi_{k}, \varphi_{k}\right\rangle & =\left\langle\psi_{k}, L \varphi_{k}\right\rangle \\
& =\int_{0}^{2 \pi} \psi_{k}\left(2 k\left(1-\frac{k^{2}}{4}\right) \psi_{k}-2 i k \psi_{k}^{\prime \prime}\right) d \theta .
\end{aligned}
$$

For $0<k<4$

$$
\int_{0}^{2 \pi} \psi_{k}^{2} d \theta=\int_{0}^{2 \pi} \psi_{k}^{\prime 2} d \theta=0
$$

This implies that $\psi_{k}=0$; thus

$$
\widehat{v}(z, \theta)=\frac{\varphi_{k}(\theta)}{(z+i(k / 2))}+\widehat{w}_{k}(z, \theta),
$$

$$
\text { for } k=1 \text { or } k=3 \text {, }
$$

where $\varphi_{k}$ is solution of problem (41).

Let $\eta$ be in $\mathbb{R}$ such that $0<\eta<1 / 2$. We note

$$
\begin{gathered}
w_{s}(t, \theta)=\frac{1}{\sqrt{2 \pi}} \int_{-\infty}^{+\infty} e^{i t z} \widehat{v}(z-i(s+2)+i \eta, \theta) d z, \\
\mu(\theta)=i \sqrt{2 \pi} e^{i t z} \widehat{v}(z, \theta) \quad \text { for fixed } t .
\end{gathered}
$$

We prove by Cauchy's theorem that

$$
v(t, \theta)=e^{(s+2-\epsilon) t} w_{s}(t, \theta)+2 i \pi \sum_{-(s+2)<\operatorname{Im}(z)<0} R_{s} .
$$

$\mu$ is an analytic function on $D_{k} ; R_{s}$ is its residue on the poles $z_{k}$. Then,

(i) for $s=-1$

$$
v(t, \theta)=e^{(1-\eta) t} w_{-1}(t, \theta)+i \sqrt{2 \pi} \psi_{1}(\theta),
$$

(ii) for $s=0$

$$
\begin{aligned}
v(t, \theta)= & e^{(2-\eta) t} w_{0}(t, \theta) \\
& +i \sqrt{2 \pi}\left(e^{3 t / 2} \psi_{3}(\theta)+e^{t / 2} \psi_{1}(\theta)+e^{t} \psi_{2}(\theta)\right),
\end{aligned}
$$

where $\psi_{i}$ are solutions of the problem (36).

Notation. Using the variables $(r, \theta)$, we introduce the following notations.

For $s=-1$, we define the two singular functions:

$$
\begin{aligned}
& \tau_{1}(r, \theta)=r^{3 / 2}\left(\sin \frac{3}{2} \theta-3 \sin \frac{\theta}{2}\right), \\
& \mu_{1}(r, \theta)=r^{3 / 2}\left(\cos \frac{3}{2} \theta-\cos \frac{\theta}{2}\right) .
\end{aligned}
$$

For $s=0$, we define four singular functions:

$$
\begin{aligned}
& \tau_{1}(r, \theta)=r^{3 / 2}\left(\sin \frac{3}{2} \theta-3 \sin \frac{\theta}{2}\right), \\
& \mu_{1}(r, \theta)=r^{3 / 2}\left(\cos \frac{3}{2} \theta-\cos \frac{\theta}{2}\right), \\
& \tau_{2}(r, \theta)=r^{5 / 2}\left(\sin \frac{5}{2} \theta-5 \sin \frac{\theta}{2}\right), \\
& \mu_{2}(r, \theta)=r^{5 / 2}\left(\cos \frac{5}{2} \theta-\cos \frac{\theta}{2}\right) .
\end{aligned}
$$


Proposition 12. For $\epsilon>0, \tau_{1}$ and $\mu_{1}$ belong to the space $H^{5 / 2-\epsilon}(V), \tau_{2}$ and $\mu_{2}$ belong to the space $H^{7 / 2-\epsilon}(V)$ and They are solutions to the problem:

$$
\begin{gathered}
\Delta^{2} \varphi=0 \quad \text { in } V, \\
\varphi=\frac{\partial \varphi}{\partial n}=0 \quad \text { on } \Gamma \cap \partial V,
\end{gathered}
$$

where $\Gamma$ is the boundary of $\Omega$.

Proof. It is the same prove that in the case $\omega=3 \pi / 2$.

For the Stokes problem we handle the case $s=-1$; thus $\mathbf{f}$ belongs to the space $\left[L^{2}(\Omega)\right]^{2}$.

Corollary 13. For $\mathbf{f}$ in $\left[L^{2}(\Omega)\right]^{2}$, the velocity is written in the form

$$
\mathbf{u}=\mathbf{u}_{r}+\mathbf{u}_{s}
$$

where $\mathbf{u}_{r}$ is in $\left[H^{2}(\Omega) \cap H_{0}^{1}(\Omega)\right]^{2}$ and there exist two real constants $\lambda$ and $\tilde{\lambda}$ such that

$$
\mathbf{u}_{s}=\lambda s_{1}+\widetilde{\lambda} \widetilde{s}_{1}
$$

with

$$
\begin{gathered}
s_{1}(r, \theta)=r^{1 / 2}\left(3 \sin \theta \sin \frac{\theta}{2}, 3(1-\cos \theta) \sin \frac{\theta}{2}\right), \\
\widetilde{s}_{1}(r, \theta)=r^{1 / 2}\left(2 \sin \frac{\theta}{2}+\sin \theta \cos \frac{\theta}{2},(1-\cos \theta) \cos \frac{\theta}{2}\right) .
\end{gathered}
$$

$s_{1}$ and $\widetilde{s}_{1}$ belong to the $\left[H^{3 / 2-\epsilon}(V)\right]^{2}$, for all $\epsilon$ positive.

For handling the singularities of the pressure we define

$$
\eta(\omega)=\inf \{\operatorname{Re}(z), z \text { is solution of }(10)\} .
$$

Indeed for $\omega \in] 0,2 \pi]$, using the Newton method, we can obtain a good approximation of roots of (10). See [20] for the approximation of $\eta(\omega)$. Thus

$$
\text { if } \pi<\omega<2 \pi, \quad 1-\frac{\pi}{\omega}<\eta(\omega)<\frac{\pi}{\omega}, \quad \eta(\omega)>\frac{1}{2} .
$$

To find the pressure, we note that $\mathbf{f}-\nu \Delta \mathbf{u}$ belongs to $\left[H^{-1}(\Omega)\right]^{2} \cap W^{\perp}$, where

$$
W=\left\{\mathbf{u} \in\left[H_{0}^{1}(\Omega)\right]^{2}, \text { such that, } \operatorname{div}(\mathbf{u})=0\right\} .
$$

Then, see [15], there exists a unique function $p$ in $L^{2}(\Omega)$ defined such that:

$$
\nabla p=\mathbf{f}+v \Delta \mathbf{u} \quad \text { in } D^{\prime}(\Omega) .
$$

From this equality we determine the singularities of the pressure from the singularities of the velocity.
Then from a regular data $\mathbf{f}$

$$
p=p_{r}+p_{s}, \quad p_{r} \in H^{1}(\Omega), \quad p_{s}=\beta S_{p 1},
$$

where $\beta$ is first singular coefficient. The linearity of (85) gives us

$$
\nabla S_{p 1}=v \Delta\left(\operatorname{curl}\left(r^{1+\eta(\omega)} \psi(\theta)\right)\right)
$$

and since we explicitly know the singularities of the velocity we can deduce, by simple integration, the singularities of the pressure.

Consider

$$
\begin{aligned}
S_{p 1}(r, \theta) & \\
& =\frac{-r^{\eta(\omega)-1}\left((1+\eta(\omega))^{2}(\partial \psi(\theta) / \partial \theta)+\left(\partial^{3} \psi(\theta) / \partial \theta\right)\right)}{(1+\eta(\omega))}
\end{aligned}
$$

in the case of crack

$$
\psi(\theta)=3 \sin \left(\frac{\theta}{2}\right)-\sin \left(\frac{3}{2} \theta\right), \quad \eta(\omega)=\frac{1}{2},
$$

in the case of $\omega=3 \pi / 2$

$$
\begin{aligned}
\psi(\theta)= & \frac{\sin ((1+\eta(\omega)) \theta) \cos (\eta(\omega) \omega)}{(1+\eta(\omega))} \\
& -\cos ((1+\eta(\omega)) \theta) \\
& +\frac{\sin ((\eta(\omega)-1) \theta) \cos (\eta(\omega) \omega)}{(1-\eta(\omega))} \\
& +\cos ((\eta(\omega)-1) \theta),
\end{aligned}
$$

and $\eta(\omega)=0.544484$.

Proposition 14. For $\epsilon>0, S_{p 1}$ belongs to $H^{0.544484}(V)$ when $\omega=3 \pi / 2$ and $S_{p 1}$ belongs to $H^{0.5}(V)$ when $\omega=2 \pi$.

Proof. It is the same proof that in the case of Proposition 3.

\section{Conclusion}

We summarize below the regularity results for the Stokes problem previously obtained.

(1) If $\mathbf{f}$ belongs to $\left[H^{s-2}(\Omega)\right]^{2}$, where $s>0$, then $(\mathbf{u}, p)$ belongs to the space $\left[H^{s}(\Omega)\right]^{2} \times H^{s-1}(\Omega)$, if $s<1+\eta(\omega)$. This means

$$
\begin{gathered}
{\left[H^{s}(\Omega)\right]^{2} \times H^{s-1}(\Omega), \quad \text { for } s<1.544484 \text { when } \omega=\frac{3 \pi}{2},} \\
{\left[H^{s}(\Omega)\right]^{2} \times H^{s-1}(\Omega), \quad \text { for } s<1.5 \text { when } \omega=2 \pi .}
\end{gathered}
$$

$(\mathbf{u}, p)$ satisfies the following stability condition:

$$
\|\mathbf{u}\|_{\left[H^{s}(\Omega)\right]^{2}}+\|p\|_{H^{s-1}(\Omega)} \leq C\|\mathbf{f}\|_{\left[H^{s-2}(\Omega)\right]^{2}} .
$$


(2) We know, from Corollaries 4 and 13 and formula (86), that if $\omega \neq 2 \pi,(\mathbf{u}, p)$ is written

$$
\mathbf{u}=\mathbf{u}_{r}+\lambda S_{1}, \quad p=p_{r}+\beta S_{p 1}
$$

and if $\omega=2 \pi$

$$
\mathbf{u}=\mathbf{u}_{r}+\lambda_{1} S_{1}+\widetilde{\lambda}_{1} \widetilde{S}_{1}, \quad p=p_{r}+\beta_{1} S_{p 1}+\widetilde{\beta}_{1} \widetilde{S}_{p 1} .
$$

If $\mathbf{f}$ belongs to $\left[H^{s-2}(\Omega)\right]^{2},\left(\mathbf{u}_{r}, p_{r}\right)$ belongs to $\left[H^{s}(\Omega)\right]^{2} \times$ $H^{s-1}(\Omega)$ for $s<1+\eta_{1}(\omega)$, where $\eta_{1}(\omega)$ is the second real solution of (10), in the band $0<\operatorname{Re}(z)<s$; hence

$$
\begin{gathered}
{\left[H^{s}(\Omega)\right]^{2} \times H^{s-1}(\Omega), \quad \text { for } s<1.908529 \text { when } \omega=\frac{3 \pi}{2},} \\
{\left[H^{s}(\Omega)\right]^{2} \times H^{s-1}(\Omega), \quad \text { for } s<2.5 \text { when } \omega=2 \pi}
\end{gathered}
$$

We have the following stability condition:

$$
\left\|\mathbf{u}_{r}\right\|_{\left[H^{s}(\Omega)\right]^{2}}+\left\|p_{r}\right\|_{H^{s-1}(\Omega)}+\left|\lambda_{1}\right|+\left|\beta_{1}\right| \leq C\|\mathbf{f}\|_{\left[H^{s-2}(\Omega)\right]^{2}} .
$$

(3) If $\mathbf{f}$ belongs to $\left[H^{s-2}(\Omega)\right]^{2}$, we can further decompose the regular part $\left(\mathbf{u}_{r}, p_{r}\right)$ of the solution as follows:

$$
\mathbf{u}_{r}=\widetilde{\mathbf{u}}_{r}+\lambda_{2} S_{2}, \quad p_{r}=\widetilde{p}_{r}+\beta_{2} \widetilde{S}_{p 2}
$$

$\left(\widetilde{\mathbf{u}}_{r}, \tilde{p}\right) \in\left[H^{s}(\Omega)\right]^{2} \times H^{s-1}(\Omega)$ for $s<1+\eta_{2}(\omega)$ where $\eta_{2}(\omega)$ is the third reel solution of $(10)$, in the band $0<\operatorname{Re}(z)<s$; then

$$
\begin{gathered}
{\left[H^{s}(\Omega)\right]^{2} \times H^{s-1}(\Omega), \quad \text { for } s<\eta_{2}\left(\frac{3 \pi}{2}\right) \text { when } \omega=\frac{3 \pi}{2},} \\
{\left[H^{s}(\Omega)\right]^{2} \times H^{s-1}(\Omega), \quad \text { for } s<3,5 \text { when } \omega=2 \pi .}
\end{gathered}
$$

$\left(\lambda_{2}, \beta_{2}\right)$ is the singular coefficient associated to the second singular function $\left(S_{2}, \widetilde{S}_{p 2}\right)$; then

$$
\begin{aligned}
& \left\|\widetilde{\mathbf{u}}_{r}\right\|_{\left[H^{s}(\Omega)\right]^{2}}+\left\|\tilde{p}_{r}\right\|_{H^{s}(\Omega)}+\left|\lambda_{1}\right|+\left|\lambda_{2}\right|+\left|\beta_{1}\right|+\left|\beta_{2}\right| \\
& \quad \leq C\|\mathbf{f}\|_{\left[H^{s-2}(\Omega)\right]^{2}} .
\end{aligned}
$$

In general we can decompose $(\mathbf{u}, p)$ as

$$
\begin{aligned}
& \mathbf{u}=\mathbf{u}_{r}+\lambda_{1} S_{1}+\lambda_{2} S_{2}+\cdots+\lambda_{k} S_{k} \\
& p=p_{r}+\beta_{1} S_{p 1}+\beta_{2} S_{p 2}+\cdots+\beta_{k} S_{p k}
\end{aligned}
$$

where $k$ is an integer number.

If $\mathbf{f}$ belongs to $\left[H^{s-2}(\Omega)\right]^{2},\left(\mathbf{u}_{r}, p_{r}\right)$ belongs to $\left[H^{s}(\Omega)\right]^{2} \times$ $H^{s-1}(\Omega)$ for $s<1+\eta_{k}(\omega)$ where $\eta_{k}(\omega), k$-ème reel solution of (10), in the band $0<\operatorname{Re}(z)<s$.

\section{Conflict of Interests}

The author declares that there is no conflict of interests regarding the publication of this paper.

\section{Acknowledgment}

This project was supported by King Saud University, Deanship of Scientific Research, College of Science Research Center.

\section{References}

[1] V. A. Kondratiev, "Boundary value problems for elliptic equations in domains with conical or angular points," Transactions of the Moscow Mathematical Society, vol. 16, pp. 227-313, 1967.

[2] P. Grisvard, Elliptic Problems in Nonsmooth Domains, vol. 24, Pitman, Boston, Mass, USA, 1985.

[3] M. Dauge, Elliptic Boundary Value Problems on Corner Domains, vol. 1341 of Lecture Notes in Mathematics, Springer, Berlin, Germany, 1988.

[4] M. Amara, C. Bernardi, and M. A. Moussaoui, "Handling corner singularities by the mortar spectral element method," Applicable Analysis, vol. 46, no. 1-2, pp. 25-44, 1992.

[5] S. Agmon, A. Douglis, and L. Nirenberg, "Estimates near the boundary for solutions of elliptic partial differential equations satisfying general boundary conditions," Communications on Pure and Applied Mathematics, vol. 12, pp. 623-727, 1959.

[6] M. Amara and M. Moussaoui, "Approximation de coefficient de singularités," Comptes Rendus de l'Académie des Sciences I, vol. 313, no. 5, pp. 335-338, 1991.

[7] I. Babuška, "Finite element method for domains with corners," Computing, vol. 6, no. 3-4, pp. 264-273, 1970.

[8] J. Lelièvre, "Sur les éléments finis singuliers," Comptes Rendus de l'Académie des Sciences A, vol. 283, no. 15, pp. 1029-1032, 1967.

[9] M. Suri, "The $p$-version of the finite element method for elliptic equations of order 2l," ESAIM, vol. 24, no. 2, pp. 265-304, 1990.

[10] G. Strang and G. J. Fix, An Analysis of the Finite Element Method, Prentice-Hall, Englewood Cliffs, NJ, USA, 1973.

[11] N. Chorfi, "Handling geometric singularities by the mortar spectral element method: case of the Laplace equation," SIAM Journal of Scientific Computing, vol. 18, no. 1, pp. 25-48, 2003.

[12] F. Ben Belgacem, C. Bernardi, N. Chorfi, and Y. Maday, "Infsup conditions for the mortar spectral element discretization of the Stokes problem," Numerische Mathematik, vol. 85, no. 2, pp. 257-281, 2000.

[13] I. Babuška, "The finite element method with Lagrangian multipliers," Numerische Mathematik, vol. 20, no. 3, pp. 179-192, 1973.

[14] O. A. Ladyzhenskaya, The Mathematical Theory of Viscous Incompressible Flow, Gordon and Breach Science Publishers, New York, NY, USA, 1962.

[15] R. Temam, Theory and Numerical Analysis of the Navier-Stokes Equations, North-Holland, Amsterdam, The Netherlands, 1977.

[16] O. Pironneau, Méthode des éléments finis pour les fluides, Masson, Paris, France, 1988.

[17] V. Girault and P. A. Raviart, Finite Element Methods for NavierStokes Equations: Theory and Algorithms, vol. 5, Springer, New York, NY, USA, 1986.

[18] L. Cattabriga, "Su un problema al contorno relativo al sistema di equazioni di Stokes," Rendiconti del Seminario Matematico della Università di Padova, vol. 31, pp. 1-33, 1961.

[19] P. Grisvard, Singularities in Boundary Value Problems, vol. 22, Springer, Amesterdam, The Netherlands, 1992.

[20] C. Bernardi and G. Raugel, "Méthodes d'éléments finis mixtes pour les équations de Stokes et de Navier-Stokes dans un polygone non convexe," Calcolo, vol. 18, no. 3, pp. 255-291, 1981. 


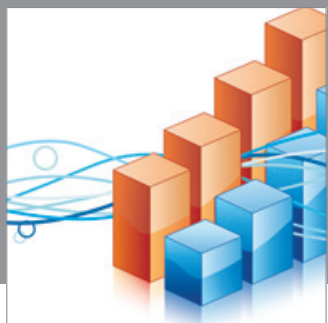

Advances in

Operations Research

mansans

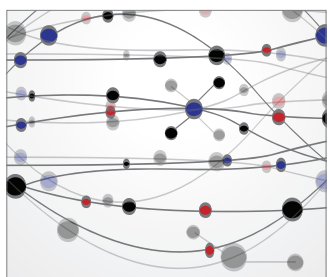

The Scientific World Journal
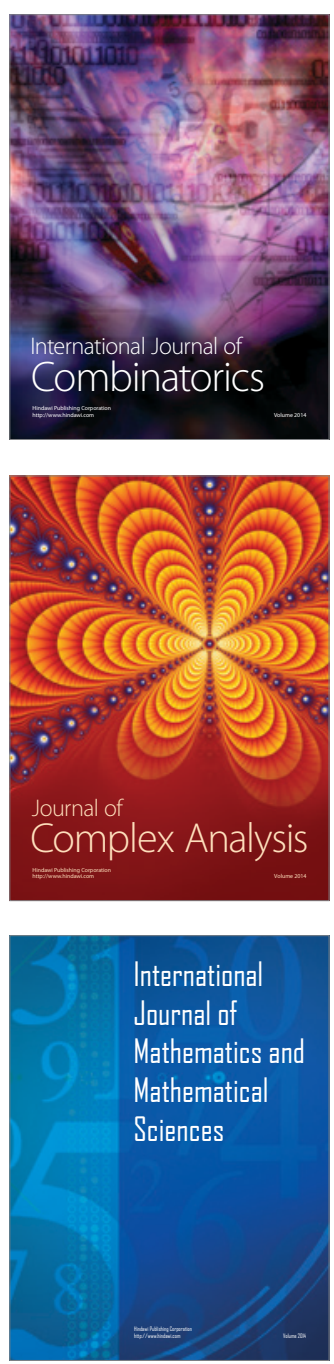
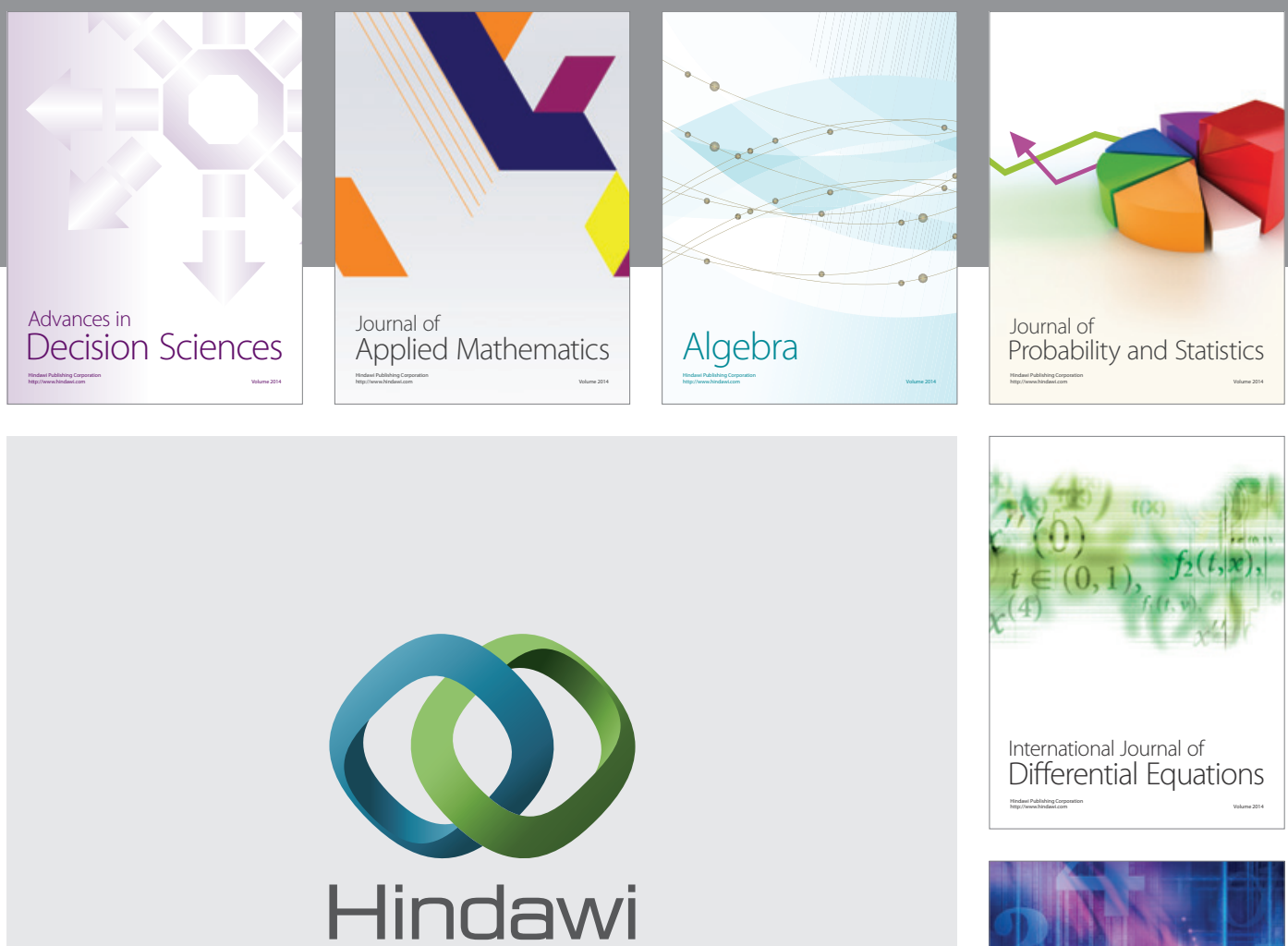

Submit your manuscripts at http://www.hindawi.com
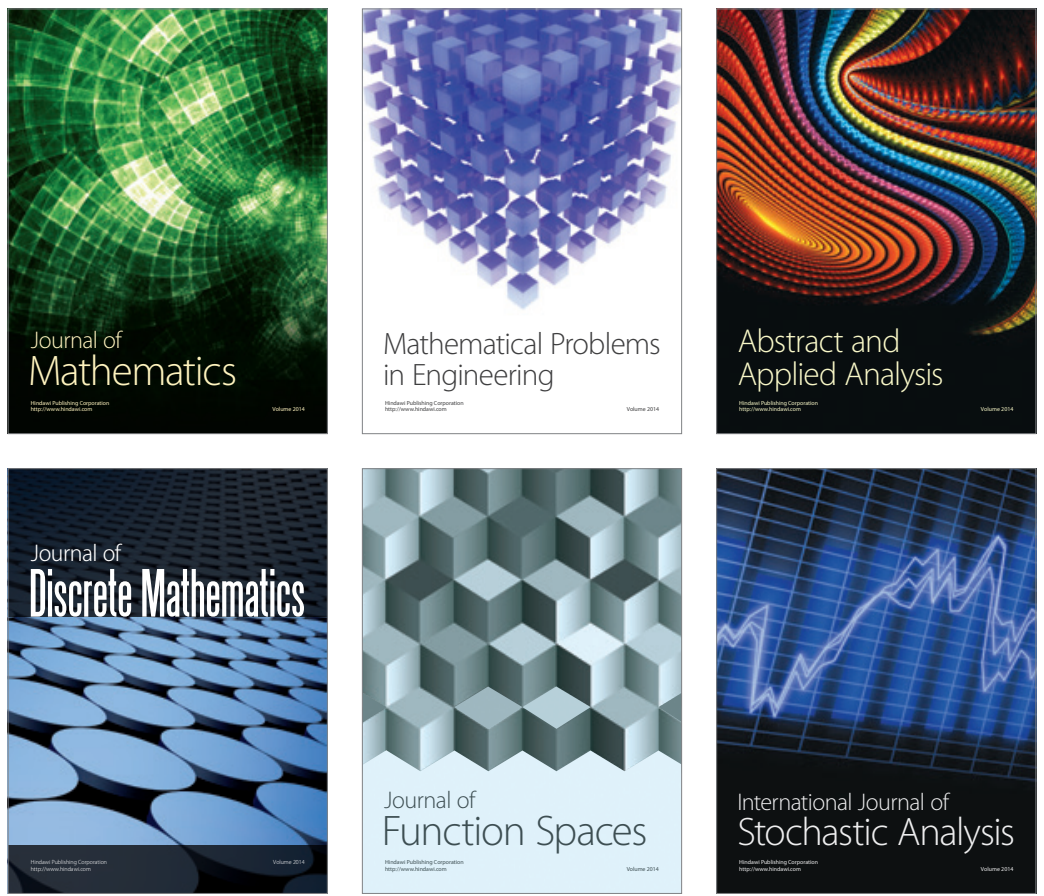

Journal of

Function Spaces

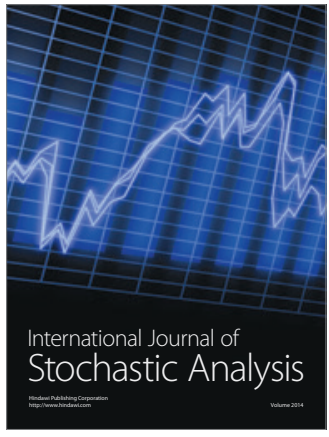

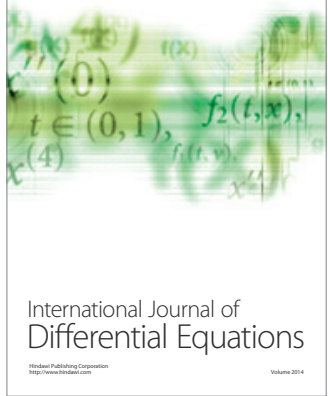
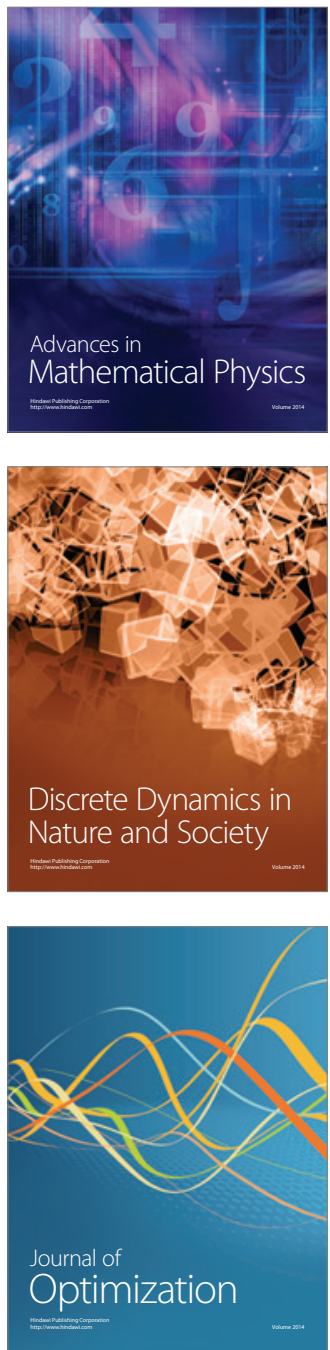